\title{
Mutation of rat Zp2 causes ROS-mediated oocyte apoptosis
}

\author{
Yan Wang ${ }^{1,2}$, Hualin Huang ${ }^{1,2}$, Minghua Zeng ${ }^{1,2}$, Ru-Ping Quan ${ }^{1,2}$, Jun-Ting Yang ${ }^{1,2}$, Dan Guo ${ }^{1,2}$, \\ Ying Sun ${ }^{1,2}$, Hongwen Deng ${ }^{3,4}$ and Hongmei Xiao(1) ${ }^{1,2}$ \\ ${ }^{1}$ Institute of Reproductive \& Stem Cell Engineering, School of Basic Medical Science, Central South University, \\ Changsha, China, ${ }^{2}$ Center of Reproductive Health, School of Basic Medical Science, Central South University, \\ Changsha, China, ${ }^{3}$ Center for Bioinformatics and Genomics, Department of Global Biostatistics and Data Science, \\ School of Public Health and Tropical Medicine, Tulane University, New Orleans, Louisiana, USA and ${ }^{4}$ Center of \\ System Biology and Data Information, School of Basic Medical Science, Central South University, Changsha, China
}

Correspondence should be addressed to H-M Xiao; Email: hmxiao@csu.edu.cn

\begin{abstract}
In this study, we investigated a gene-edited $\left(\mathrm{Zp} 2^{\mathrm{MT} / \mathrm{MT}}\right)$ rat model of infertility caused by the failure to express the zona pellucida glycoprotein 2 (ZP2) due to the significant reduction of mRNA amount. We examined the defects in the zona pellucida (ZP) caused by ZP2 nullification and the influence of these defects on aspects of oocyte development, including apoptosis and fertilization ability. To investigate the cause of the influence to the oocytes' development, we evaluated the morphology of follicular transzonal projections (TZPs), known as 'bridges', which mediate the bidirectional signaling between the oocyte and surrounding granulosa cells and the level of reactive oxygen species (ROS) in ovulated eggs. Our results showed that two types of ZP defects were generated in the $Z p 2^{\mathrm{MT} / \mathrm{MT}}$ rat,that is, ZP intact but thinned and ZP cracked (or even absent). The fertilization rate of the ovulated eggs reduced in both types, while increased oocyte apoptosis was observed only in the latter type. Moreover, the increased oocyte apoptosis rate correlated closely with the reduction in follicular TZPS and increased ROS levels in ovulated egg. In conclusion, nullification of rat ZP2 destroyed the integrity of the ZP, impaired the bidirectional signaling between the oocyte and surrounding granulosa cells. Therefore, the resulting infertility likely occurs via elevation of oxidative stress and oocytes apoptosis.

Reproduction (2020) $160353-365$
\end{abstract}

\section{Introduction}

Zona pellucida (ZP) is a unique shield-like structure enclosing the growing oocyte, ovulated egg, and preimplantation embryos (Wassarman 2008). ZP plays a vital role in oogenesis (Wassarman \& Litscher 2012), fertilization (Gupta et al. 2012) and preimplantation embryogenesis (Suzuki et al. 1995, Lamas-Toranzo et al. 2019). In mammals, the ZP is formed by three to four zona pellucida glycoproteins (ZPGs) depending on the species, for example, four in human and rat (designated hZP1-4 and rZP1-4, respectively) and three in mouse (mZP1-3). Since we reported the first familial infertility caused by human $Z P 1$ mutation in 2014 (Huang et al. 2014), an increasing number of $Z P$ mutations-associated infertile cases has been reported (Chen et al. 2017, Liu et al. 2017, Dai et al. 2019a,b, Sun et al. 2019, Zhou et al. 2019). One of these cases, hZP2-associated infertility, is characterized by intact and thinned ZP and in vitro fertilization (IVF) failure, although the number of retrieved eggs (16-25 eggs per cycle) was undiminished (Dai et al. 2019b). However, Zp2 null mice had unsustainable, extremely thinned ZP during folliculogenesis, producing fewer ovulated eggs
(1.4 per mouse) with no ZP (Rankin et al. 2001). The ZP phenotype and the damage to ovulation in humans affected by hZP2-associated infertility are much less severe than those in the $Z p 2$ null mouse. Furthermore, in humans, ZP4 can possibly compensate for the loss of ZP2 (Dai et al. 2019b). Therefore, we hypothesized that the existence of ZP4 in rat makes this a better choice as an animal model of hZP2-associated infertility.

To investigate the details of hZP2-associated infertility, we used the CRISPR/Cas9 system to create a gene-edited rat model carrying a similar $Z p 2$ mutation, that is, the $Z p 2^{\mathrm{MT} / M T}$ rat. However, the phenotype of the $Z p 2^{\mathrm{MT} / M T}$ rat was unexpected in that most antral follicular oocytes and ovulated eggs had cracked or even absent ZPs and presented apoptotic morphology. The transzonal projections (TZPs) are filamentary structures consisting of elongated cytoplasmic tips spanning the ZP to connect the oocyte and surrounding granulosa cells. These 'bridges' mediate the bidirectional signaling that is critical for the survival and development of oocytes and granulosa cells (Simon et al. 1997, Wang et al. 2019). Previously, we suggested that destruction of the integrity of the ZP could reduce the density of TZPs and decrease the quality of eggs (Wang et al. 2019). Several previous 
studies have indicated that reduction of oocyte-granulosa cell bidirectional signaling may trigger the generation of reactive oxygen species (ROS) and that the resulting increase in oxidative stress is one of the major factors that induce oocyte apoptosis (Tiwari et al. 2015). Thus, we hypothesize that, in the $Z p 2^{\mathrm{MT} / \mathrm{MT}}$ rat, destruction of the integrity of the ZP impairs the bidirectional signaling between the oocyte and surrounding granulosa cells, elevates oxidative stress, and induces oocyte apoptosis, and thus it results in female infertility. To test this hypothesis, we investigated the fertility of the $\mathrm{Zp} 2^{\mathrm{MT} / \mathrm{MT}}$ rat, as well as apoptosis of follicular oocytes, the morphology of TZPs in folliculogenesis, and the level of ROS in ovulated eggs. To the best of our knowledge, this is the first study to explore the mechanism of oocyte apoptosis caused by ZP gene mutations.

\section{Materials and methods}

\section{Animals}

Zp2 $2^{\mathrm{MT} / \mathrm{MT}}$ rats were generated by CRISPR-Cas9 technologymediated DNA editing. To make the mutation in rats comparable to those of humans affected by ZP2-associated infertility (c.1695_2A>G and c.1691_1694dup), two guide RNAs (gRNA), gRNA 1 (5'-TATCCTACCAACGGCCTTATAGG-3') and gRNA 2 (5'-GGTGCTGTGGCTGCACTAGTGGG-3'), were designed based on bioinformatic analysis to introduce sitespecific double-strand breaks (DSB) in exon14 and exon18 of rat $Z p 2$, respectively (Fig. $1 \mathrm{~A}$ ). Recombination of genomic DNA was completed by non-homologous end-joining (NHEJ) (Fig. 1A). The gRNAs and Cas9 mRNA were transcribed and purified in vitro using MEGAshortscript ${ }^{\mathrm{TM}} \mathrm{T} 7$ Transcription Kit (AM1354, Thermo Fisher Scientific), MEGAclear TM Transcription Clean-Up Kit (AM1908, Thermo Fisher Scientific), and Cas9 plasmid (CAS9P, Sigma-Aldrich). The purified gRNAs and Cas9 mRNA were co-injected into the zygotes collected from Sprague-Dawley rats by oviduct flushing $13 \mathrm{~h}$ post-mating. After in vitro culture in the KSOM medium (MR-101-D, SigmaAldrich) at $37^{\circ} \mathrm{C}$ and $5 \% \mathrm{CO}_{2}$ overnight, two-cell embryos were obtained and transferred into the oviducts of pseudo-pregnant rats. Approximately 3 weeks later, neonates were genotyped by PCR using three pairs of primers (Fig. $1 \mathrm{~A}$, arrows or arrow heads) and Universal Genomic DNA Extraction Kit (9765, TaKaRa), and PCR Amplification Kit (M712B, Promega). Only the rats with the pre-designed mutation were considered as the F0 generation and were used to establish a strain. F0 rats were

Table 1 Antibodies, primers and gRNAs.

\begin{tabular}{|c|c|c|c|c|c|c|c|}
\hline \multirow[b]{2}{*}{ Resources } & \multirow[b]{2}{*}{ Names } & \multirow[b]{2}{*}{ Description } & \multirow[b]{2}{*}{ Manufacturer } & \multirow[b]{2}{*}{ Catalog } & \multicolumn{3}{|c|}{ Other information } \\
\hline & & & & & $\mathrm{IHC}$ & WB & Product length, bp \\
\hline \multicolumn{2}{|c|}{ Primary antibodies } & Rabbit-derived & & & & & \\
\hline & ZP1 & & $\begin{array}{l}\text { Santa-cruz, } \\
\text { USA }\end{array}$ & 134757 & $1: 200$ & & \\
\hline & ZP2 & & Abcam, USA & ab2719677 & $1: 200$ & $1: 500$ & \\
\hline & ZP3 & & $\begin{array}{l}\text { Santa-cruz, } \\
\text { USA }\end{array}$ & 25802 & $1: 200$ & & \\
\hline & ZP4 & & Abcam, USA & ab2553903 & $1: 200$ & & \\
\hline $\begin{array}{r}\text { Secondary } \\
\text { antibody }\end{array}$ & ZP1-4 & Goat Anti-Rabbit IgG & Affinity, USA & S0001 & $1: 200$ & $1: 2000$ & \\
\hline \multicolumn{8}{|c|}{ Primers $\left(5^{\prime}-3^{\prime}\right)$} \\
\hline & Pair 1 & & & BioSune, China & & & 316 \\
\hline & $\mathrm{F}$ & AGGATGACCGTGAGATGC & & & & & \\
\hline & $\mathrm{R}$ & AACCTATGATGACTGCTGAG & & & & & \\
\hline & Pair 2 & & & - & & & 490 \\
\hline & $\mathrm{F}$ & TTCCAGGATGACCGTGAGATGC & & & & & \\
\hline & $\mathrm{R}$ & CAAGCAACCCTGGTGTGTAGGAGTA & & & & & \\
\hline & Pair 3 & & & & & & 294 \\
\hline & $\mathrm{F}$ & CAGAATAGGAGAACGGGAGG & & & & & \\
\hline & $\mathrm{R}$ & GTAAGGTTCAATTCCTGATTGG & & & & & \\
\hline & Pair 4 & & & & & & $\begin{array}{l}Z p 2^{\text {WTMTT }} \text { rats: } 2108 ; \\
\text { Zp } 2^{\text {MT/MT }} \text { rats: } 1706\end{array}$ \\
\hline & $\mathrm{F}$ & AGGTATCTTCCAACATGGCTAGG & & & & & \\
\hline & $\mathrm{R}$ & GTCCAATCAGGAATTGAACCTTAC & & & & & \\
\hline \multicolumn{8}{|c|}{ 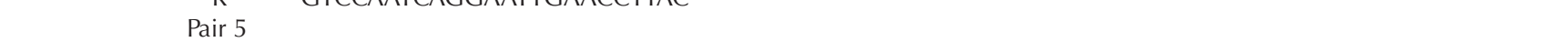 } \\
\hline & $\mathrm{F}$ & GGGCTTTGGACAAAGGAACA & & & & & 189 \\
\hline & $\mathrm{R}$ & AGCATGTCCСCTTGGAAGTT & & & & & \\
\hline & Pair 6 & & & & & & 150 \\
\hline & $\mathrm{F}$ & CCСATCTATGAGGGTTACGC & & & & & \\
\hline & $\mathrm{R}$ & TTTAATGTCACGCACGATTTC & & & & & \\
\hline \multicolumn{2}{|c|}{$\begin{array}{l}\text { gRNA (PAM in bold; } \\
\left.5^{\prime}-3^{\prime}\right)\end{array}$} & & - & - & & & $\begin{array}{l}\text { Obtained from } \\
\text { in vitro transcription }\end{array}$ \\
\hline & gRNA 1 & TATCCTACCAACGGCCTTATAGG & & & & & \\
\hline & gRNA 2 & GGTGCTGTGGCTGCACTAGTGGG & & & & & \\
\hline
\end{tabular}

F, forward; PAM, protospacer adjacent motif; R, reverse. 
crossed with WT females to obtain $Z p 2^{\mathrm{WT} / \mathrm{MT}} \mathrm{F} 1$ rats, which were intercrossed to obtain F2 rats $\left(Z p 2^{\mathrm{WT} / \mathrm{WT}}, \mathrm{Zp} 2^{\mathrm{WT} / \mathrm{MT}}\right.$ and $\left.\mathrm{Zp} 2^{\mathrm{MT} / \mathrm{MT}}\right)$. F3 rats were obtained by intercrossing $\mathrm{F} 2$ rats.

In this study, the $Z p 2^{\mathrm{WT} / \mathrm{NT}}$ and $Z p 2^{\mathrm{MT} / \mathrm{MT}}$ rats were F2 or $\mathrm{F} 3$, whereas the $Z p 2^{\mathrm{WT} / \mathrm{MT}}$ rats were F1. Only sexually mature rats (aged 13-15 weeks) were used. All rats were housed in an environmentally controlled room maintained at $23 \pm 1{ }^{\circ} \mathrm{C}$ with a $12 \mathrm{~h}$ light:12 h darkness cycle. Animal protocols were approved by The Animal Care and Use Committee, Central South University, China.

\section{$D N A, m R N A$ and protein verification}

DNA verification was performed in all the F1 rats. Following the manufacturers' instructions, gemonic DNA was extracted (9765, TaKaRa) from rat tail samples and Zp2 gene was amplified (M712B, Promega) by PCR using the primers (Pair 1-3. Pair 1-forward: 5'-AGGATGACCGTGAGATGC-3', upstream of the target deletion DNA fragment, Pair 1-reverse: 5'-AACCTATGATGACTGCTGAG-3', downstream of the above fragment; Pair 2-forward: 5'-TTCCAGGATGACCGTGAG
ATGC-3', upstream of the fragment, Pair 2-reverse: 5'-CAAGCAACCCTGGTGTGTAGGAGTA-3', within the fragment; Pair 3-forward: 5'-CAGAATAGGAGAACGGGAGG-3', within the fragment, Pair 3-reverse: 5'-GTAAGGTTCAAT TCCTGATTGG-3' ${ }^{\prime}$, downstream of the fragment) described in Fig. $1 \mathrm{~A}$ and Table 1. After purification, DNA sequencing was performed. In addition, off-target analysis was carried out by examining the top ten potential off-target sites (predicted by CRISPOR, shown in Supplementary file, see section on supplementary materials given at the end of this article) of gRNA 1 and gRNA 2.

RNA was extracted from rat ovary samples according to the manufacturer's protocol of TRIzol ${ }^{\text {TM }}$ Plus RNA Purification Kit (12183555,ThermoFisherScientific).RT ofmRNAwasperformed with the use of GoScript ${ }^{\mathrm{TM}}$ RT System (A5001, Promega). PCR amplification of Zp2 cDNA was performed with a pair of primers (forward: 5'-AGGTATCTTCCAACATGGCTAGG-3', reverse: 5'-GTCCAATCAGGAATTGAACCTTAC-3', Table 1), which covered the whole coding sequence. The lengths and sequences of PCR products were confirmed by agarose gel electrophoresis and Sanger sequencing, respectively.

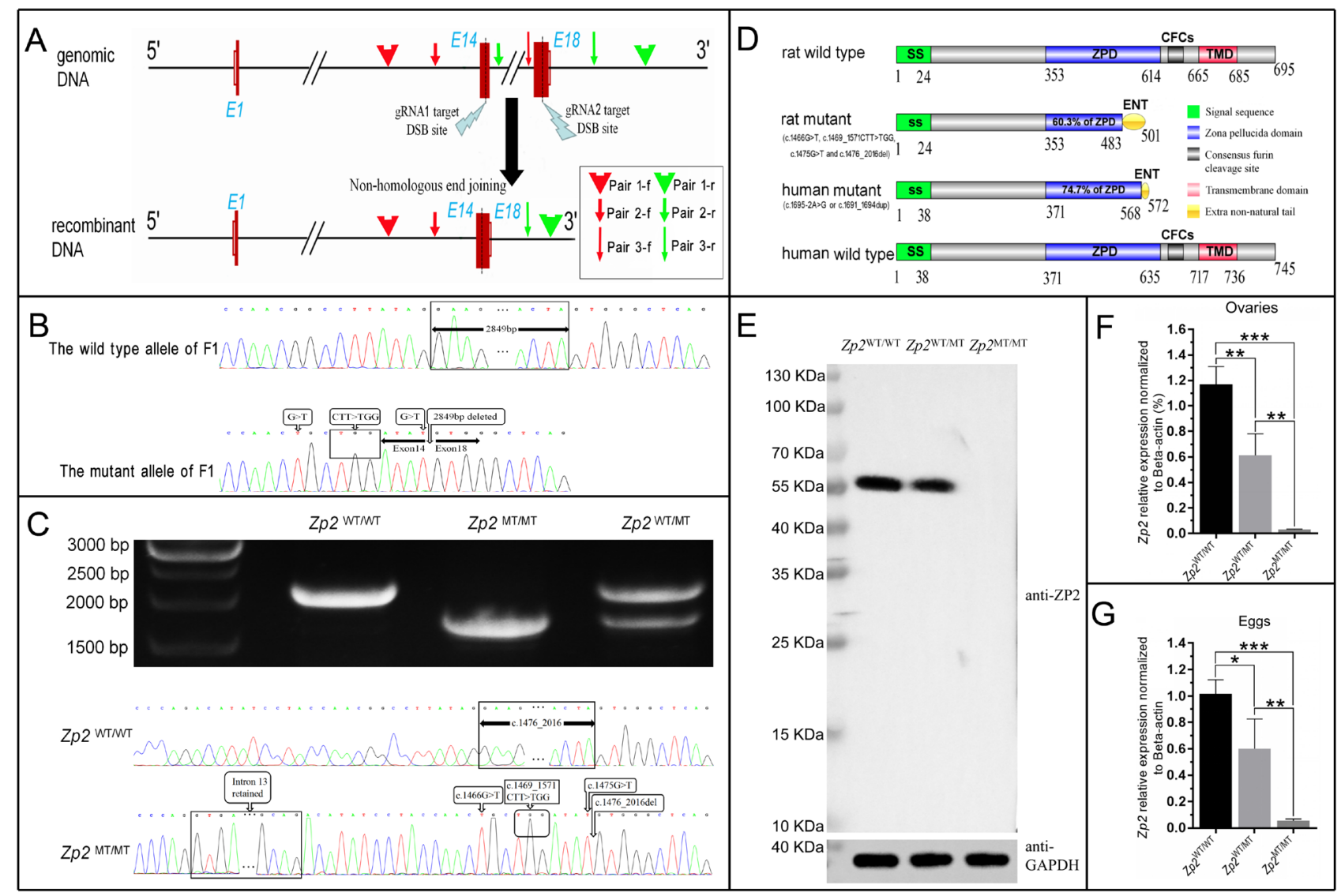

Figure 1 Target mutations in rat $Z p 2$ gene caused intronic retention and protein nullification due to drastic reduction of mRNA amount. (A) The scheme for generation and genotyping of $Z p 2^{\mathrm{MT} / \mathrm{MT}}$ rats. Solid bars indicate exons and hollow bars indicate untranslated regions. Paired primers for genotyping are indicated by arrows or arrow heads. E: exon; f: forward; r: reverse; DSB, double-strand breaks. (B) Genomic DNA verification. (C) Messenger RNA verification by agarose gel electrophoresis (upper) and Sanger sequencing (lower). (D) Bioinformatic alignment of human and rat mutant ZP2 proteins predicted by mRNA sequences. (E) Protein verification by Western blot analysis. (F and G) Relative quantification of Zp2 mRNA by qPCR using ovary tissue samples (F) or ovulated egg samples (G). ${ }^{*} P<0.05 ;{ }^{* *} P<0.01 ;{ }^{* * *} P<0.001$. 
Subsequently, quantitative PCR (qPCR) was performed with two pairs of primers (Pair 5 for Zp2 and Pair 6 for Actb. Pair 5-forward: 5'-GGGCTTTGGACAAAGGAACA-3', Pair 5-reverse: 5'-AGCATGTCCCCTTGGAAGTT-3'; Pair 6-forward: 5'-CCCATCTATGAGGGTTACGC-3', Pair 6-reverse: 5'-TTTAATGTCACGCACGATTTC-3'. Table 1) according to the manufacturer's instructions of MonAmp ${ }^{\mathrm{TM}}$ ChemoHS qPCR Mix (MQ00201S, Monad, China) and the products were sequenced. In addition, in each genotype, we used 3-5 normal ovulated eggs for qPCR, following the manufacturer's protocol of Fast Advanced Cells-to-CTTM qPCR Kit (A35379, Thermo Fisher Scientific).

Proteins were verified by Western blot analysis. The total proteins were extracted from ovary tissue with cell lysis kit (87787, Thermo Fisher Scientific) and separated by sodium dodecyl sulfate PAGE (SDS-PAGE). Proteins were transferred to the nitrocellulose membrane and incubated with the ZP2 primary antibody (ab2719677, abcam) at $4{ }^{\circ} \mathrm{C}$ overnight. The membrane was then washed and incubated with the secondary antibody (S0001, affinity, USA.) at room temperature (RT) for $1 \mathrm{~h}$. After washing, the ZP2 bands on the membrane were visualized by enhanced chemiluminescent detection reagent kit (34577, Thermo Fisher Scientific).

\section{Isolation of ovaries and ovulated eggs}

Female rats were killed by intraperitoneal injection of pentobarbital sodium with the dosage of $200 \mathrm{mg} / \mathrm{kg}$ body weight, and their ovaries were isolated directly without hormone stimulation. Immediately after isolation, the ovaries were frozen in the liquid nitrogen for mRNA sequencing, qPCR, and Western blotting, or fixed in 4\% paraformaldehyde solution for periodic-acid Schiff (PAS) staining, immunohistochemistry, terminal deoxynucleotidyl transferase 2'-deoxyuridine, 5 '-triphosphate nick-end labeling (TUNEL) analysis and phalloidin staining.

For super-ovulation, female rats were intraperitoneally injected with $40 \mathrm{IU}$ pregnant mare serum gonadotropin (PMSG) between $5 \mathrm{pm}$ and $6 \mathrm{pm}$. After $48 \mathrm{~h}$, they were injected with 40 IU human chorionic gonadotropin (hCG). Fifteen hours later, these rats were killed and their oviducts were isolated and placed into the paraffin oil (pre-incubated at $37^{\circ} \mathrm{C}$ and $5 \% \mathrm{CO}_{2}$ for $1 \mathrm{~h}$ ) of a culture dish containing some oil-covered droplets. These droplets were classified as M2-hyaluronidase droplets, composed of M2 medium (M7167, Sigma-Aldrich) and hyaluronidase $(1 \mathrm{mg} / \mathrm{mL})$ or $\mathrm{M} 2$-alone droplets, composed of M2 medium alone. Under the microscope, the expanded ampullae were torn open using two $30 \mathrm{G}$ hypodermic needles and the overflowing cumulus-oocyte complexes (COCs) were drawn into the M2-hyaluronidase droplets. The COCs were gently blown with a pipette with a very fine blunt tip to disperse the granulosa cells. When the eggs were almost completely detached from the granulosa cells, they were transferred into the $\mathrm{M} 2$-alone droplets. After thorough washing with $\mathrm{M} 2$ medium, the eggs were viewed under a microscope (IX73, Olympus Corporation). For each rat, the ovulated eggs were counted and their morphology was evaluated. The eggs with normal morphology (full-grown spherical oocyte with homogeneous cytoplasm) were ready for IVF, qPCR, and ROS analysis.

\section{Ovarian histology}

After fixation in the 4\% paraformaldehyde solution for 12-16 $\mathrm{h}$, ovaries were dehydrated with a series of ethanol solutions with increasing concentrations $(30 \%, 50 \%, 70 \%, 90 \%$, and $100 \%)$. Then the ovaries were embedded in paraffin for PAS staining, immunohistochemistry, and TUNEL analysis, or in the optimal cutting temperature compound (6404, Newcomer Supply, USA) for phalloidin staining which requires frozen sections. Consecutive sections were prepared for immunohistochemistry, whereas intermittent sections were made at an interval of $200 \mu \mathrm{m}$ for PAS staining, TUNEL analysis, and phalloidin staining. The tissue section thickness was $4 \mu \mathrm{m}$ for PAS staining and TUNEL analysis and $8 \mu \mathrm{m}$ for phalloidin staining. Before use, all paraffin sections were thoroughly dewaxed by dimethylbenzene, rehydrated by a series of ethanol solutions with decreasing concentrations (100\%, 90\%, 70\%, 50\%, 30\%), and then washed with double distilled water and PBS solution.

PAS staining was performed according to the manufacturer's protocol of PAS Stain Kit (D004-1-2, Nanjing Jiancheng Bioengineering Institute, China). The growing follicles (GFs) were classified as follows: primary follicles (PFs), with only one intact layer of cubic granular cells; secondary follicles (SFs), with two (early secondary follicles, ESFs) or more (late secondary follicles, LSFs) intact layers of cubic granulosa cells but without any obvious antrum; and antral follicles (AFs), with an obvious antrum. The classification was based on the principle suggested by Gook et al. (2008), but herein some modifications were made, for example, SFs were classified into ESFs and LSFs. According to the description of Escobar et al. (2012), the oocytes in the GFs were considered to be morphological dead when at least one of the following conditions was met: severe deformation; fragmentation; or diffuse vacuolation. In each section, the GFs were classified and counted to calculate the constituent ratios of the total GFs in the $Z p 2^{\mathrm{WT} / \mathrm{NT}}$ and $Z p 2^{\mathrm{MT} / \mathrm{MT}}$ rats. The morphology of GFs was evaluated and the differences between $Z p 2^{\mathrm{WT} / \mathrm{WT}}$ and $Z p 2^{\mathrm{MT} / \mathrm{MT}}$ rats in terms of apoptosis were analyzed.

For immunohistochemistry, antigen retrieval was performed using the microwave heating method as following: ovarian tissue sections were put into $1 \mathrm{~L}$ of boiled sodium citrate antigen retrieval solution (C1031, Solarbio, China); the solution was reboiled for $4 \mathrm{~min}$ and then rested for $6 \mathrm{~min}$; the step was repeated three times and finally the solution cooled down naturally. After incubation with the primary antibody (ZP1: 134757, santa-cruz; ZP2: ab2719677, abcam; ZP3: 25802, santa-cruz; ZP4: ab2553903, abcam) at the dilution of 1:200 overnight, the tissue sections were washed five times with PBS and then were incubated with the secondary antibody (S0001, affinity) at $37^{\circ} \mathrm{C}$ for $1 \mathrm{~h}$. Subsequently, sections were thoroughly washed and stained with hematoxylin. For negative controls, the same procedures were used except that the primary antibody was replaced with PBS. TUNEL assays were performed according to the manufacturer's instructions of In Situ Cell Death Detection Kit (11684795910, Roche) and phalloidin staining was performed as described by Wang et al. (2019). Finally, sections were stained with DAPI to indicate the cell nucleus. With the utilization of image-pro plus 6.0 software (Media Cybernetics Corporation, USA), the density 

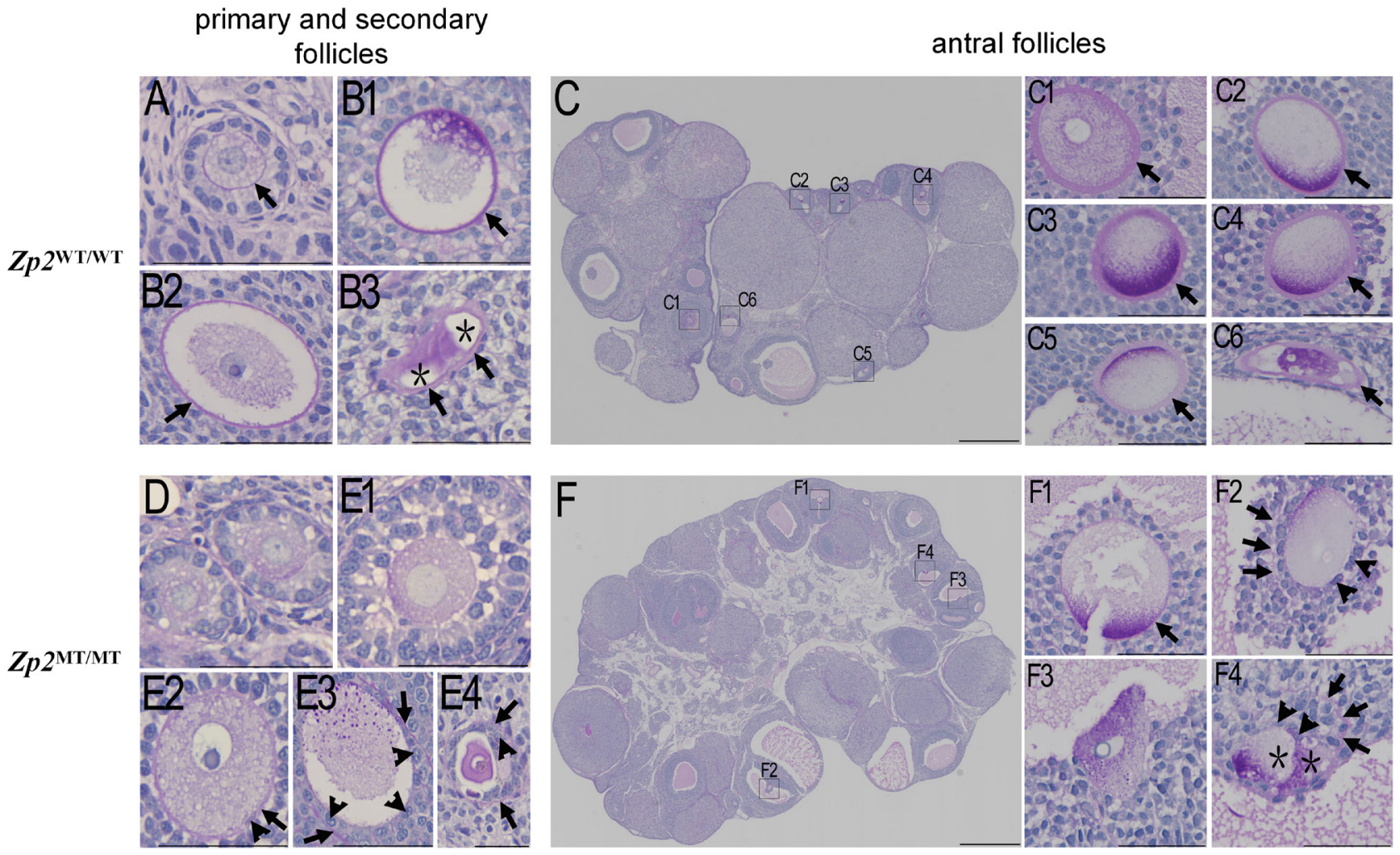

Figure 2 Follicular ZP defects and accompanied oocyte dysmorphology in Zp2 $2^{\mathrm{MT} / \mathrm{MT}}$ rats. A and D indicate primary follicles; B1-3 and E1-4 indicate secondary follicles; $\mathrm{C}(\mathrm{C} 1-6)$ and $\mathrm{F}(\mathrm{F} 1-4)$ indicate antral follicles. Arrows indicate the ZP and arrow heads indicate the ectopic granulosa cells. Asterisks indicate the fragments of dead oocytes. Scale bar: $1 \mathrm{~mm}$ in C and F, $50 \mu \mathrm{m}$ in others.

of TZPs in each GFs was calculated according to the following formula:

density of TZPs $=\frac{\text { integral optical density }(\mathrm{IOD}) \text { within the ZP area }}{\text { the area of } \mathrm{ZP}\left(\mu \mathrm{m}^{2}\right)}$

\section{In vitro experiments of ovulated eggs}

For IVF, male rats were killed and their epididymides were isolated. The semen was expelled into a sterile culture dish using fine tweezers. Using a glass capillary with a fine, curved and sealed tip, a small amount of semen was collected and put into the oil-covered droplets of $100 \mu \mathrm{L}$ human tubal fluid (HTF) solution (MR-070-D, Sigma-Aldrich), which was preequilibrated at $37^{\circ} \mathrm{C}$ under $5 \% \mathrm{CO}_{2}$ for $8-12 \mathrm{~h}$. After $30 \mathrm{~min}$, a certain volume of the HTF solution (containing the sperm) was transferred into a new pre-equilibrated oil-covered HTF droplet $(100 \mu \mathrm{L}$, not containing sperm) to create an IVF droplet with a final sperm density of $10^{6} / \mathrm{mL}$. Three hours later and immediately after isolation, eggs were transferred into the IVF droplets and co-incubated with the capacitated sperm for $6 \mathrm{~h}$. The eggs were then thoroughly washed with HTF solution and then cultured for $4 \mathrm{~h}$. Under the relief contrast microscope (IX73, Olympus), zygotes with two pronuclei were counted and the fertilization rates were calculated.

The ROS level of eggs was assessed with the ROS assay kit (CA1410, Solarbio, China) according to the manufacturer's instructions. Then $2^{\prime}, 7^{\prime}$-dichlorodihydrofluorescein diacetate (DCFH-DA) was added into the M2-alone droplet containing the isolated eggs, and the final concentration of DCFH-DA was controlled at $10 \mu \mathrm{mol} / \mathrm{L}$. In vitro culture of eggs was continued for $30 \mathrm{~min}$, during which time the eggs were gently blown with a fine pipette every $10 \mathrm{~min}$. The eggs were then washed five times with M2 medium and imaged under a fluorescence microscope.

\section{Fertility assessment}

The litter size of each female was recorded after mating with a male (aged 13-30 weeks) proven to be fertile for 3 months or until a pregnancy was observed. During the 3 months of observation, females that did not become pregnant were considered infertile.

\section{Statistical analysis}

The measurement data were shown as mean \pm S.D., and the differences among groups were analyzed by one-way ANOVA following the Student-Newman-Keuls test. The counting data were shown as rate \pm S.E., and the differences between groups were analyzed by Chi-square test. All tests were performed with the software SPSS 19. $P<0.05$ was considered to indicate statistical significance. 


\section{Results}

The Zp2 ${ }^{M T / M T}$ rat was successfully generated, but the mutant ZP2 protein failed to be expressed due to the significant reduction of $m R N A$ amount

A total of 97 zygotes were micro-injected and transferred into pseudo-pregnant rats, resulting in the delivery of four pups. Only one male (F0) carried the pre-designed mutations, consisting of a 2849 bp deletion between exon 14 and exon 18 and five adjacent point mutations upstream of the deletion in both $Z p 2$ alleles. These mutations were also confirmed in the mutant alleles of $\mathrm{Zp2}$ in all six F1 rats (two males and four females) (Fig. 1B). Off-target analysis of gRNAs for all the F1 rats showed that all the tested genomic DNA sequences (Supplementary file, site 1-10 and site 11-20 were the top 10 potential off-target sequences for gRNA1 and
gRNA2, respectively) were normal with no mutations. The four F1 females and six randomly selected F2 females ( $3 Z p 2^{\text {WTMT }}$ and $3 Z p 2^{\text {MT/MT }}$ ) were used for mRNA and protein analyses. In addition to the five point mutations (c.1466G $>$ T, c.1469_1571CTT>TGG, c. $1475 \mathrm{G}>\mathrm{T})$ in the mutant $Z p 2 \mathrm{mRNA}$, a deletion of 541 bp (c.1476_2016) was identified between exon14 and exon18, as well as retention of the complete intron 13 (Fig. 1C). This intronic retention led to a premature termination codon (PTC) in the intron 13 and resulted in a truncated protein which lacked 212 amino acids at the C-terminal. Although comparable with the mutant ZP2 protein in humans affected by ZP2-associated infertility based on bioinformatics (Fig. 1D), the mutant ZP2 protein in rats was undetectable by Western blot analysis (Fig. $1 \mathrm{E})$. For each genotype, three F2 generation rats were used for qPCR analysis, with three biological replicates

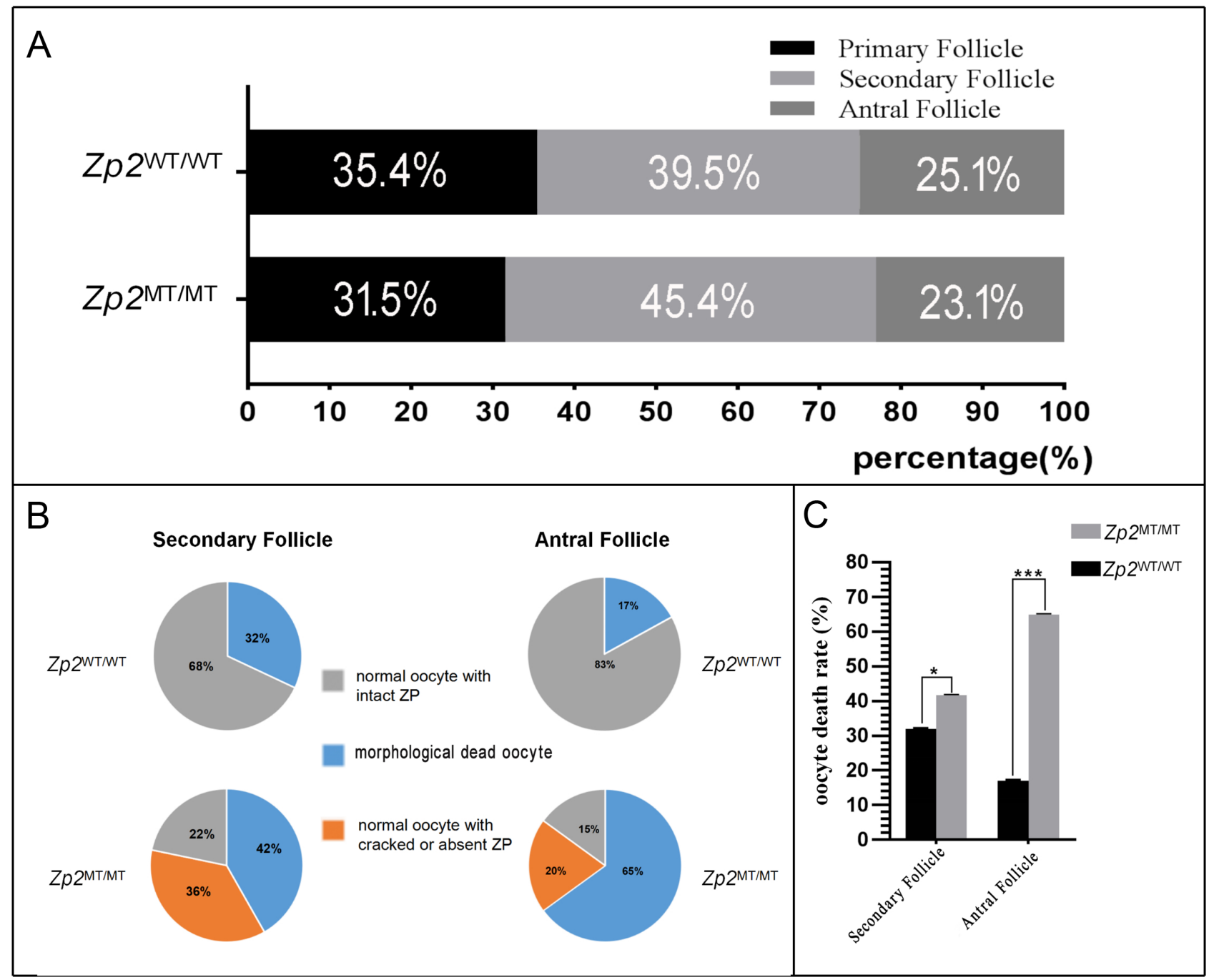

Figure 3 Normal constituent ratios and increased oocyte apoptosis of growing follicles in Zp $2^{\mathrm{MT} / \mathrm{MT}}$ rats. (A) Constituent ratios of growing follicles. (B) Constituent ratios of secondary (left) and antral (right) follicles. (C) Oocyte death rates of secondary and antral follicles based on morphological evaluation. ${ }^{*} P<0.05 ;{ }^{* * *} P<0.001$. 


\section{$Z p 2^{\mathrm{WT} / \mathrm{WT}} \quad Z p 2^{\mathrm{MT} / \mathrm{MT}}$}

\section{negative control}

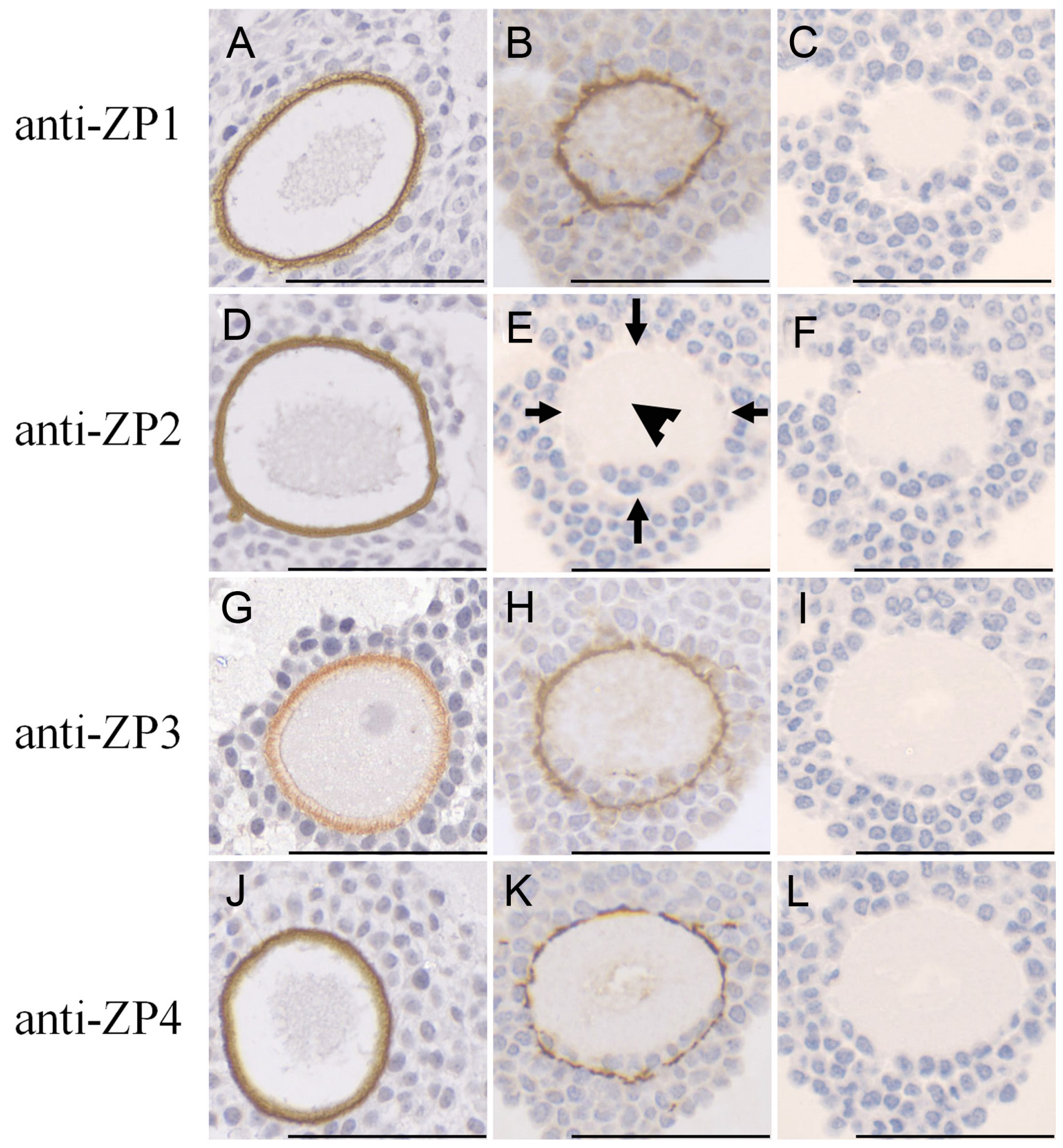

Figure 4 Absence of ZP2 expression while other ZPGs are normally expressed in Zp2 $2^{\mathrm{MT} / \mathrm{MT}}$ rats. The absence of a ZP2 signal in the ZP or cytoplasm is indicated by the arrows or arrow head, respectively. Scale bar: $50 \mu \mathrm{m}$. 
Table 2 Number of ovulated eggs and live births.

\begin{tabular}{|c|c|c|c|c|}
\hline & \multicolumn{4}{|c|}{ Ovulated eggs } \\
\hline & \multirow[b]{2}{*}{ Dead } & \multicolumn{2}{|c|}{ Live } & \multirow[b]{2}{*}{ Live births } \\
\hline & & With intact ZP & With cracked or absent ZP & \\
\hline 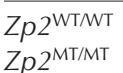 & $\begin{array}{l}0.38 \pm 0.74(8)^{*} \\
8.50 \pm 2.42(10)\end{array}$ & $\begin{array}{r}21.75 \pm 5.34 \\
2.00 \pm 0.67\end{array}$ & $\begin{array}{l}0.00 \pm 0.00 \\
1.60 \pm 1.07\end{array}$ & $\begin{array}{r}11.43 \pm 2.23(7)^{\#} \\
0.00 \pm 0.00(10)\end{array}$ \\
\hline
\end{tabular}

${ }^{*}$ Mean \pm S.D. (number of animals); ${ }^{*}$ Mean \pm S.D. (number of litters).

for each rat. The results of qPCR analysis suggested an obvious decline in $Z p 2 \mathrm{mRNA}$ levels in the $Z p 2^{\mathrm{WT} / \mathrm{MT}}$ and $Z p 2^{\mathrm{MT} / M T}$ rats, regardless of the samples, that is, ovaries (Fig. 1F) or ovulated eggs (Fig. 1G). Notably, the decline in $Z p 2^{\mathrm{MT} / \mathrm{MT}}$ rats was for more dramatic than that in the $Z p 2^{\text {WT/MT }}$ rats (Fig. $1 \mathrm{~F}$ and G). Taken together, these findings suggested the reduction of mRNA amount resulting in the failure of mutant ZP2 protein expression.

\section{Two types of oocytes were observed in the GFs of $\mathrm{Zp2}{ }^{\mathrm{MT} / M T}$ rats, one with intact but thinned $Z P$, another with cracked or no ZP}

A total of 24 ovaries from 12 rats (six $Z p 2^{\text {WTTWT }}$ and six $Z p 2^{\mathrm{MT} / \mathrm{MT}}$ ) were isolated. For each ovary, four intermittent ovarian tissue sections were stained with PAS. The ZP was stained red in GFs (Fig. 2, arrows). In the $Z p 2^{\mathrm{WT} / W T}$ rats, most PFs contained a distinguishable and intact ZP (Fig. 2A), which was thickened and much more conspicuous in the ESFs (Fig. 2B1) and LSFs (Fig. $2 \mathrm{~B} 2$ ). However, in the $Z p 2^{\mathrm{MT} / \mathrm{MT}}$ rats, the $\mathrm{ZP}$ was absent in all the PFs (Fig. 2D) and the majority of ESFs (Fig. 2E1), although an intact ZP was present in each LSF. This observation indicated a delay in ZP formation in the $Z p 2^{\mathrm{MT} / M T}$ rats. Two types of growing oocytes were observed in the LSFs of $Z p 2^{\mathrm{MT} / \mathrm{MT}}$ rats: one with intact ZP, the other with cracked ZP (Fig. 2E2 and E3). The severity of ZP cracking varied greatly. In some LSFs, the cracking of the ZP was very slight and only a few granulosa cells were ectopic in the perivitelline space (PVS), some of which contacted the oocytes directly instead of via the TZPs (Fig. 2, the arrow head in E2). In other LSFs, the ZP cracking was severe and multiple granulosa cells were ectopic (Fig. 2E3). In the Zp2 $2^{\mathrm{WT} / \mathrm{WT}}$ rats, the $\mathrm{ZP}$ in AFs was intact and thick (Fig. $2 \mathrm{C} 1-5$ ). In the $Z p 2^{\mathrm{MT} / \mathrm{MT}}$ rats, there were two types of oocytes in the AFs: one with intact but extremely thinned ZP (Fig. 2F1), the other with cracked (Fig. 2F2 and F4) or even absent (Fig. 2F3) ZP. Similar to the LSFs, the cracking of the ZP in the AFs of

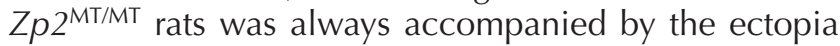
of the granulosa cells (Fig. 2, arrow heads in F2 and F4). In both $Z p 2^{\mathrm{WT} / \mathrm{NT}}$ and $Z p 2^{\mathrm{MT} / \mathrm{MT}}$ rats, no morphological characteristics of death were observed in oocytes in the PFs, but in some SFs (Fig. 2B3 and E4) and AFs (Fig. 2C6 and F3-4), the oocytes presented obvious morphological characteristics of death.

\section{In the Zp2 ${ }^{M T / M T}$ rats, morphological characteristics of death of oocytes were significantly increased in SFs and particularly in AFs}

In total, 96 ovarian tissue sections were analyzed for folliculogenesis (48 Zp2 $2^{\mathrm{WT} T \mathrm{NT}}$ and $48 \mathrm{Zp} 2^{\mathrm{MT} / \mathrm{MT}}$ ). The total number of GFs in $Z p 2^{\mathrm{MT} / M T}$ rats was 628 , which was comparable with that in the Zp2 $2^{\text {WTNT }}$ rats $(n=681)$. Moreover, there was no significant difference in the constituent ratios of GFs between the $Z p 2^{\text {WTMT }}$ and $Z p 2^{\mathrm{MT} / \mathrm{MT}}$ rats (Fig. 3A; $P=0.097$ ). This result indicated that folliculogenesis of $Z p 2^{\mathrm{MT} / \mathrm{MT}}$ rats was not affected quantitatively. However, in terms of quality, oocytes showing morphological characteristics of death accounted for the largest proportion, whereas ZP-intact oocytes accounted for the smallest proportion in both the SFs and AFs of $Z p 2^{\mathrm{MT} / \mathrm{MT}}$ rats (Fig. 3B). Notably, the opposite results were observed in the $Z p 2^{\text {WTNTT }}$ rats (Fig. 3B). Furthermore, in the $Z p 2^{\mathrm{MT} / \mathrm{MT}}$ rats, the proportions of oocytes showing morphological characteristics of death were significantly increased in both SFs and particularly in AFs, in which the oocytes showing morphological characteristics of death (Fig. 2C6 and F3-4) accounted for 65\% in $\mathrm{Zp} 2^{\mathrm{MT} / \mathrm{MT}}$ rats in contrast to only $17 \%$ in the $Z p 2^{\text {WTMTT }}$ rats (Fig. $3 \mathrm{C}$ ).

\section{The ZP of Zp2 ${ }^{M T / M T}$ rats was composed of only ZP1, $Z P 3$, and ZP4, while the mutant ZP2 protein was absent}

Each immunohistochemistry analysis was performed three times. In the $Z p 2^{\text {WTNWT }}$ rats, all the four ZPGs (ZP1-4) were successfully detected in the ZP (Fig. 4A, $D, G$ and J). However, in the $Z p 2^{M T / M T}$ rats, only three ZPGs (ZP1, ZP3, and ZP4) were successfully detected in the ZP (Fig. 4B, H and K). Notably, the mutant ZP2 was completely undetectable, indicating its absence in both the ZP (Fig. 4, arrows in E) and the cytoplasm of oocytes (Fig. 4, arrow head in E). This result indicated the absence of the expression of the mutant ZP2 protein in the $Z p 2^{\mathrm{MT} / M T}$ rats, which was consistent with the results of Western blot analysis.

\section{In the Zp2 ${ }^{M T / M T}$ rats, most ovulated eggs exhibited morphological characteristics of death, and the rest presented two types: one with intact $Z P$, the other with cracked or no ZP}

In total, 298 ovulated eggs were collected (177 from $8 Z p 2^{\text {WTNTT }}$ rats and 121 from $10 Z p 2^{\mathrm{MT} / M T}$ rats) 
(Table 2). In the Zp2 ${ }^{\text {WTNWT }}$ rats, most eggs (98.3\%) were morphologically normal and only three eggs were fragmented and considered to be dead (Fig. 5A, B, left and Table 2). In contrast, most eggs (70.3\%) from the Zp $2^{\mathrm{MT} / \mathrm{MT}}$ rats showed morphological characteristics of death (Fig. $5 \mathrm{~A}, \mathrm{~B}$, right and Table 2) including flattened, coarse, and deformed structure and even severe shrinkage. The severity of shrinkage differed among the eggs, with some eggs showing slight shrinkage (Fig. 5A, crossed arrow I), whereas others were drastically reduced in size (Fig. $5 \mathrm{~A}$, crossed arrow II) or even completely unobservable (Fig. 5A, crossed arrow III). The ZP phenotype of these morphologically dead eggs also differed greatly, with some greatly expanded and thinned (Fig. 5A, arrow $1)$, while others were cracked with obviously severed tips and normal in size (Fig. 5A, arrow 2) or absent (Fig. 5A, arrow 3). In the PVS of the dead eggs, ectopia of granulosa cells was consistently observed (Fig. 5A, arrow heads). These results indicated that all the dead eggs in the $Z p 2^{\mathrm{MT} / M T}$ rats originated from GFs with cracked or absent ZP. Although the number was small, some eggs in the $Z p 2^{\mathrm{MT} / \mathrm{MT}}$ rats survived (Fig. $5 \mathrm{~B}$, right and Table 2), showing normal cellular morphology (Fig. $5 \mathrm{~A}$, asterisks). These eggs were classified into two types: one with intact but thinned ZP (Fig. 5A, arrow 4) and another with cracked or absent ZP (Fig. 5A, arrow 5). All these live eggs from the $Z p 2^{\mathrm{MT} / \mathrm{MT}}$ rats, as well as 129 live eggs from $Z p 2^{W T W T}$ rats, were fertilized in vitro. The fertilization rates of both types of eggs from $Z p 2^{\mathrm{MT} / M \mathrm{MT}}$ rats were much lower than that of the eggs from $Z p 2^{\text {WT/NT }}$ rats (Fig. 5C and D).

\section{In the Zp2 ${ }^{\mathrm{MT} / M T}$ rats, only the eggs with a cracked or no ZP showed significantly increased apoptosis, which was closely correlated to the reduction in TZPs and increased ROS levels}

A total of 45 ovary tissue sections (15 from $3 Z p 2^{\text {WTTWT }}$ rats and 30 from $6 \mathrm{Zp} 2^{\mathrm{MT} / \mathrm{MT}}$ rats) were analyzed by the TUNEL assay. Only the oocytes in AFs with a clear germinal vesicle were analyzed (Fig. 6A). There were 17 such oocytes in the $Z p 2^{\text {WTMT }}$ rats and 29 in the $Z p 2^{\text {MT/MT }}$ rats ( 7 with intact ZP and 22 with cracked or absent ZP). There was no significant difference in the apoptosis rates of the oocytes in Zp2 ${ }^{\mathrm{WT} W \mathrm{WT}}$ rats and the ZP-intact oocytes in the $Z p 2^{\mathrm{MT} / \mathrm{MT}}$ rats $(P=1.000)$, whereas the apoptosis rate of oocytes with cracked or absent ZP was much higher ( $P=0.003$ ) (Fig. 6B). For the TZPs analysis, five ovary tissue sections from three rats of each genotype were subjected to phalloidin staining. In the GFs of the $Z p 2^{\text {WTNTT }}$ rats, as well as in the ZP-intact GFs of $Z p 2^{\mathrm{MT} / \mathrm{MT}}$ rats, the TZPs were abundant and usually laid perpendicularly to the oocyte surface (Fig. 6A, white arrow heads). However, in some ZP-cracked GFs of $Z p 2^{\mathrm{MT} / M T}$ rats, the TZPs were very sparse and laid in a disorderly manner (Fig. 6A, white arrow heads). The GFs with abnormal TZPs were in the majority, particularly in the ZP-cracked GFs with multiple ectopic granulosa cells in the PVS (Fig. 6A, black arrow heads). These changes in TZPs were pervasive, rather than confined to the region of ectopic granulosa cells (Fig. 6A). For statistical analysis, 13 GFs from Zp2 $2^{\text {WTNTT }}$ rats and 21 GFs from $Z p 2^{\mathrm{MT} / \mathrm{MT}}$ rats (including $11 \mathrm{ZP}$-intact GFs and 10 ZP-cracked GFs) were included for the calculation of TZPs' density. In the ZP-intact GFs, the density of TZPs was comparable $(P>0.05)$ with that in the GFs of $Z p 2^{\mathrm{WT} /}$ WT rats, but in the ZP-cracked GFs, the density of TZPs was significantly reduced $(P<0.001)$ (Fig. $6 \mathrm{C})$. For ROS analysis, 45 eggs from five $Z p 2^{\text {WTTWT }}$ rats and 24 eggs from six $Z p 2^{\mathrm{MT} M \mathrm{MT}}$ rats (14 ZP-intact eggs and ten eggs with cracked or absent $Z \mathrm{P}$ ) were evaluated. There was no significant difference in the IOD of ZP-intact eggs in the $Z p 2^{\mathrm{MT} / \mathrm{MT}}$ rats and that of ovulated eggs in the $Z p 2^{\mathrm{WT} / \mathrm{NT}}$ rats $(P=0.493)$ (Fig. $6 \mathrm{D})$. However, the average IOD of the eggs with cracked or no $Z P$ in the $Z p 2^{\mathrm{MT} / \mathrm{MT}}$ rats was significantly higher than that of eggs in the $Z p 2^{\mathrm{WT} /}$ ${ }^{W T}$ rats $(P<0.001)$ (Fig. 6D). This indicated a significant increase in ROS levels in the eggs with cracked or no ZP, but not in the eggs with intact $Z \mathrm{P}$, in the $Z p 2^{\mathrm{MT} / \mathrm{MT}}$ rats.

\section{The $\mathrm{Zp} 2^{\mathrm{MT} / \mathrm{MT}}$ female rats were infertile}

The litter sizes of seven $Z p 2^{\text {WTNTT }}$ females and ten $Z p 2^{\mathrm{MT} /}$ MT females were recorded (Table 2). Every $Z p 2^{\text {WT }}$. female produced a litter with the average litter size of 11.43 (Table 2). However, none of the $Z p 2^{\mathrm{MT} / \mathrm{MT}}$ females became pregnant and no pups were produced (Table 2 ).

\section{Discussion}

In this study, we described a ZP2-null rat strain characterized by extreme vulnerability of the ZP, severe apoptosis of the oocytes, and absolute loss of fertility. Most importantly, our results indicate that the increased ROS levels associated with the destruction of ZP integrity and reduction in TZPs may contribute to the oocyte death. This result indicates a detrimental, even lethal consequence in the metabolism of the oocyte caused by the perforation or absence of the ZP due to mutations in $Z p 2$ and the resulting protein nullification. It is still unknown that by which means $Z p 2$ mutation caused ZP2 nullification. However, considering that $Z p 2$ mutation results in drastic mRNA reduction, the nonsense mediated mRNA decay, a RNA surveillance system that ensures accelerated degradation of PTCcontaining mRNA to avoid the synthesis of truncated proteins with potentially deleterious effects for cells (Lambert et al. 2020) may be a plausible explanation. These findings highlight the importance of ZPGs coding genes on the survival of oocytes during folliculogenesis and provide novel insights in the potential mechanism, especially from the aspect of ZP2 gene and its functions. 


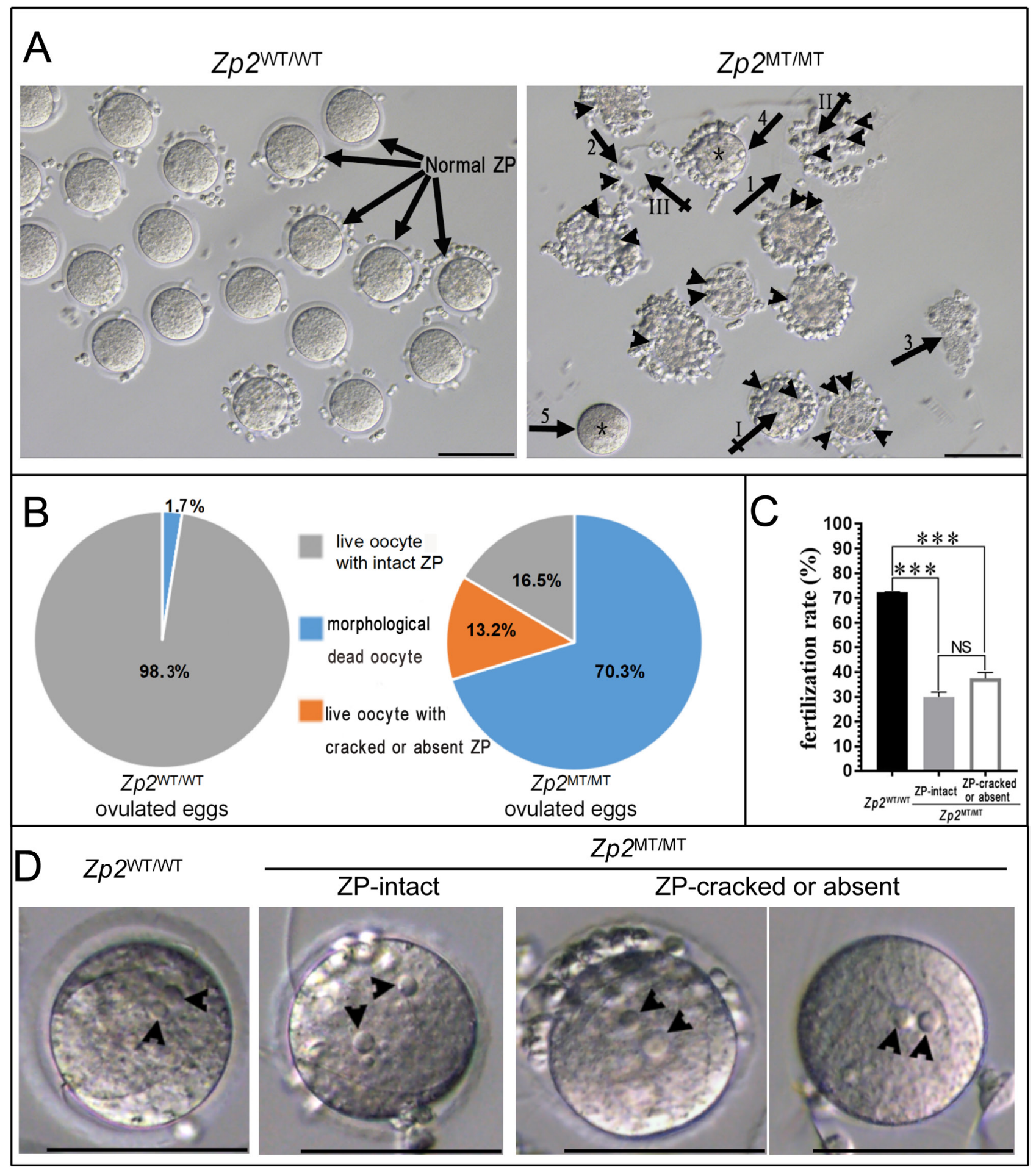

Figure 5 Abnormalities in the morphology and fertilizing ability of ovulated eggs in Zp2 ${ }^{\mathrm{MT} / \mathrm{MT}}$ rats. (A) Morphology of ovulated eggs. Arrows indicate the ZPs: arrow 1, the cracked, extremely expanded and thinned ZP; arrow 2, the cracked ZP with obviously severed tips, but unexpanded; arrow 3, the absence of the ZP in a morphologically dead oocyte; arrow 4, the intact but thinned ZP; arrow 5, the absence of the ZP in a morphologically normal oocyte. Crossed arrows indicate the shrinkage of oocytes: crossed arrow I, slight shrinkage; crossed arrow II, extreme shrinkage; crossed arrow III, the oocyte was not visible. Arrow heads indicate the ectopic granulosa cells, and asterisks indicate the morphologically normal oocytes. Scale bar: $100 \mu \mathrm{m}$. (B) Constituent ratios of ovulated eggs based on morphological evaluation. (C) Fertilization rates of ovulated eggs in vitro. ${ }^{* * *} P<0.001$; NS: not significant. (D) Representative images for fertilized eggs. Arrow heads indicate the pronucleus. Scale bar: $100 \mu \mathrm{m}$. 


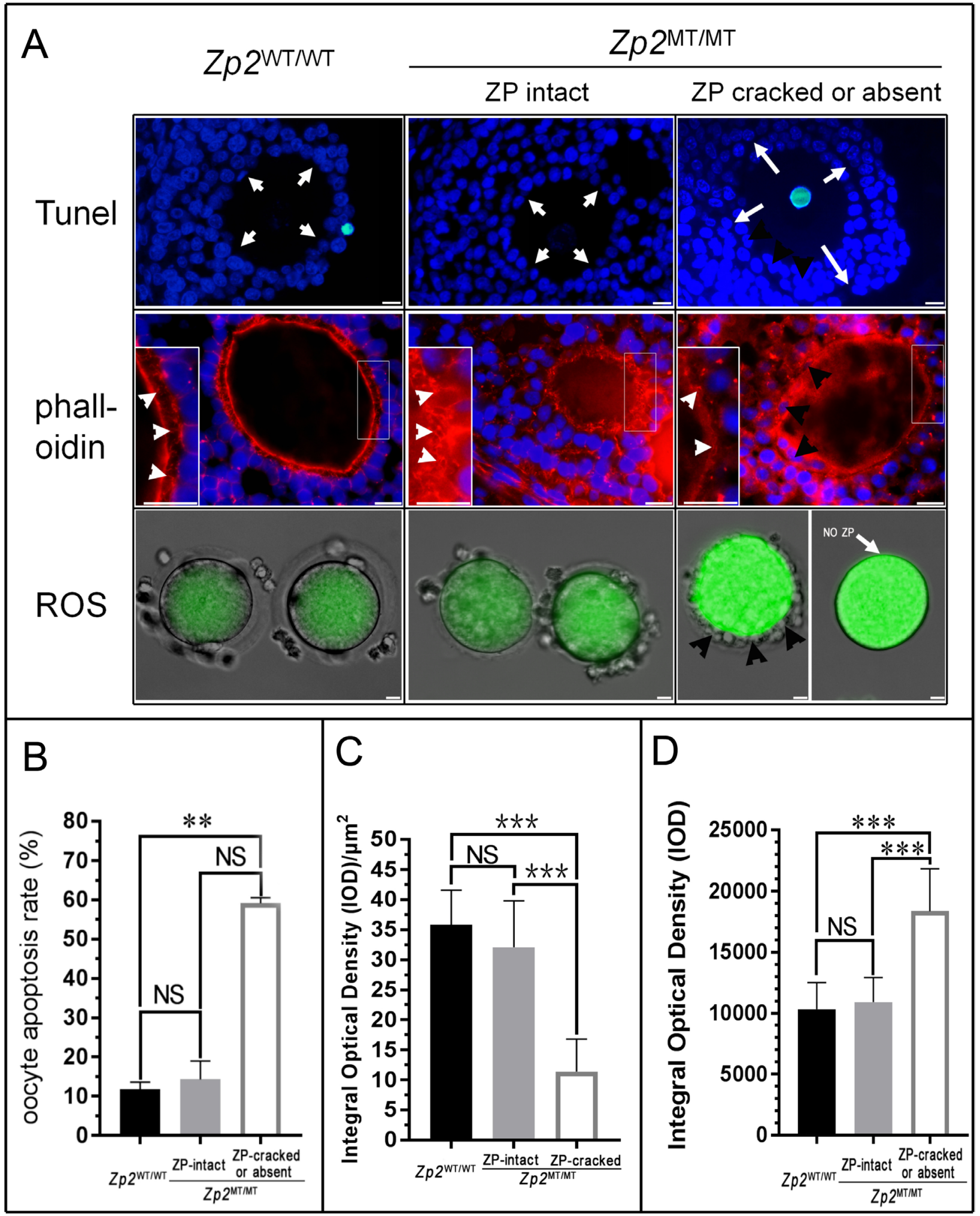

Figure 6 Abnormalities in the oocytes' apoptosis and ROS level as well as the TZPs' density in Zp2 ${ }^{\mathrm{MT} / M T}$ rats. (A) Representative images of TUNEL assay, phalloidin staining, and ROS level analysis. The degree of TUNEL assay positivity is shown in yellow-green. White arrows indicate the locations of ZPs, white arrow heads indicate the filamentary TZPs, and black arrow heads indicate the ectopic granulosa cells. Scale bar: $10 \mu \mathrm{m}$. (B) Apoptosis rates of oocytes in antral follicles based on the TUNEL assay. ${ }^{* *} P<0.01$; NS: not significant. (C) Densities of TZPs in growing follicles. ${ }^{* * *} P<0.001$; NS: not significant. (D) ROS levels of ovulated eggs based on integral optical density analysis. ${ }^{* * *} P<0.001 ;$ NS: not significant. 
Although loss of ZP2 function always leads to infertility, the severity of the ZP defect (evaluation based on the existence and integrity of eggs' ZP: ZP-free is severest, ZP-cracked is intermediate, and ZP-intact is slightest) varies in different species, with the ZP2-null rat (some eggs are ZP-free, the others are ZP-cracked or ZP-intact) exhibiting intermediate severity compared with the ZP2-null mouse (all the eggs are ZP-free) and the ZP2-truncated human (all the eggs are ZP-intact). Avella et al. reported that the thickness and robustness of the $\mathrm{ZP}$ in ZP2-null mouse were restored by the expression of hZP4, suggesting that hZP4 can compensate for the absence of mZP2 (Avella et al. 2014). According to amino acid sequences alignments, hZP4 and rZP4 show similar rates of coverage on their respective ZP2 (49\% and $54 \%$, respectively), but hZP4 has a higher identity (to ZP2) rate $(40.1 \%)$ than rZP4 $(33.8 \%)$, supporting a stronger putative compensatory ability of hZP4 compared with that of rZP4. In addition, although both containing ZP4, the human ZP is twice as thick as that in the rat. This observation may suggest that, compared with rZP4, hZP4 plays a much more important role in increasing the ZP thickness. Taken together, these findings indicate that the ZP defect in ZP2-truncated humans is less severe than that in ZP2-null rats because hZP4 can better compensate for hZP2 and that the ZP2-null mouse, which lacks ZP4, has the most severe ZP defect.

Previous studies have clarified a view that only the ZP-intact eggs can contribute to pups production and that the ZP-cracked and ZP-free eggs are eliminated during preimplantation development in vivo because of the poor or no embryo protection (Rankin et al. $1999,2001)$. The major reason for the infertility of the ZP2-null rat and mouse is the drastic reduction in the number of ovulated eggs, especially the ZP-intact eggs. However, the number of ovulated eggs in ZP2-truncated humans is not reduced. The ZP phenotypes indicate that the integrity of the ZP is the key factor for preventing the impairment of oogenesis. This phenomenon is also supported by studies in ZP3-null mice or ZP4-null rabbits (Liu et al. 1996, Wassarman et al. 1997, LamasToranzo et al. 2019). In mice lacking a single Zp3 allele, or rabbits lacking both ZP4 alleles, the ZP is thinned but still intact and the number of ovulated eggs is not reduced (Wassarman et al. 1997, Lamas-Toranzo et al. 2019). In mice lacking both $Z p 3$ alleles, the ZP is absent throughout folliculogenesis and the numbers of antral follicles and ovulated eggs are significantly reduced (Liu et al. 1996). Additionally, in the ZP1-null mouse, a few of the growing follicles are ZP-cracked, which leads to the reduction in the numbers of $\mathrm{AF}$ and ovulated eggs, although these effects are not as severe as those in the ZP3-null mouse (Rankin et al. 1999). Similarly, the number of eggs (without degeneration) retrieved from all the 11 human beings affected by reported infertilities associated with cracked or absent ZP due to mutations in ZPGs coding genes are universally lessened (averagely 0-8.5 eggs per cycle depending on the patients, 1.4 eggs per cycle as a whole) (Huang et al. 2014, Chen et al. 2017, Liu et al. 2017, Dai et al. 2019a, Sun et al. 2019).

Cracks in ZP are always accompanied by ectopia of the granulosa cells in the perivitelline space, which leads to direct contact of the oocyte and surrounding granulosa cells rather than through the TZPs. Previous studies have implicated granulosa cell ectopia as the initiator of the developmental impairment of the oocyte mediated by changes in its metabolism, leading to alterations in the density and distribution of TZPs, which may be exacerbated by a form of vicious circle by further changing the oocyte's metabolism (Wang et al. 2019). This putative positive feedback explains the pervasive nature of the decline in follicular TZPs in the $\mathrm{Zp} 2^{\mathrm{MT} / \mathrm{MT}}$ rat, rather than an effect that is confined to the area of ectopic granulosa cells. Although the metabolic changes may be complicated, the results are clearly detrimental and even often lethal to the oocytes. Oocyte degeneration or apoptosis associated with the destruction of the integrity of the ZP has been reported in cases of human infertility caused by ZPGs coding genes, although the mechanism remains unclear (Chen et al. 2017, Sun et al. 2019). ROS may play a critical role, and although the mechanism underlying the accumulation of ROS remains to be elucidated, some clues have been provided by previous studies. Pandey et al. suggested that decreased levels of cyclic 3', 5'-AMP (cAMP), an important signaling molecule that is transferred from the granulosa cells to oocytes through TZPs, are closely related to the increased ROS levels (Pandey \& Chaube 2014). However, it is not practicable to detect the egg's cAMP level in the $Z p 2^{\text {MT/MT }}$ rat because of the drastic decreased number of survived eggs. The increased ROS may cause oocyte damage by contributing to activation of the mitochondria-mediated apoptotic pathway (Tiwari et al. 2015).

It can be speculated that the altered metabolic changes described previously also contribute to the reduced fertilization rate of the $Z p 2^{\mathrm{MT} / \mathrm{MT}}$ rat eggs with cracked or absent ZP. However, the Zp2 ${ }^{\mathrm{MT} M T}$ rats' ZP-intact eggs also show a reduced fertilization rate which cannot be attributed to the metabolic change only. Notably, because of the failure of sperm binding, the eggs of ZP2-truncated humans cannot be fertilized unless intracytoplasmic sperm injection (ICSI) is performed. This indicates a vital role of hZP2 in sperm recognition and binding. Strikingly, the ZP-intact eggs of $Z p 2^{\mathrm{MT} / \mathrm{MT}}$ rats recognize and bind the sperm effectively, indicating a much less important role of rZP2 than hZP2 in fertilization. This might make the ZP-intact eggs of $Z p 2^{\mathrm{MT} / \mathrm{MT}}$ rat fertilizable, although the fertilization ability remains impaired. 


\section{Supplementary materials}

This is linked to the online version of the paper at https://doi. org/10.1530/REP-20-0037.

\section{Declaration of interest}

The authors declare that there is no conflict of interest that could be perceived as prejudicing the impartiality of the research reported.

\section{Funding}

This study was supported by National Key R\&D Program of China (2017YFC1001100 and 2016YFC1201805), National Natural Science Foundation of China (81471453 and 81501248), Natural Science Foundation of Hunan Province of China (2015JJ2166 and 2017JJ3425), and construction project of the center of reproductive health, Central South University (164990007). HW Deng was partially supported by grants from the NIH (R01-AR069055, U19-AG055373, R01-MH104680, R01-AR059781, and P20-GM109036) and Edward G Schlieder Endowment fund at Tulane University.

\section{Author contribution statement}

$\mathrm{HX}$ designed the research. $\mathrm{Y} W, M \mathrm{Z}$, and $\mathrm{H} \mathrm{H}$ performed the experiments. Y W and R-P Q analyzed the data. J-T Y, D G, and $Y$ S contributed to discussion. $Y W$ wrote the paper. $\mathrm{H} \mathrm{X}$ and $H D$ reviewed and revised the paper. $H$ D contributed to finalization of the paper.

\section{References}

Avella MA, Baibakov B \& Dean J 2014 A single domain of the ZP2 zona pellucida protein mediates gamete recognition in mice and humans. Journal of Cell Biology 205 801-809. (https://doi.org/10.1083/jcb.201404025)

Chen T, Bian Y, Liu X, Zhao S, Wu K, Yan L, Li M, Yang Z, Liu H, Zhao H et al. 2017 A recurrent missense mutation in zp3 causes empty follicle syndrome and female infertility. American Journal of Human Genetics 101 459-465. (https://doi.org/10.1016/j.ajhg.2017.08.001)

Dai C, Chen Y, Hu L, Du J, Gong F, Dai J, Zhang S, Wang M, Chen J, Guo J et al. 2019a ZP1 mutations are associated with empty follicle syndrome: evidence for the existence of an intact oocyte and a zona pellucida in follicles up to the early antral stage. A case report. Human Reproduction 34 2201-2207. (https://doi.org/10.1093/humrep/dez174)

Dai C, Hu L, Gong F, Tan Y, Cai S, Zhang S, Dai J, Lu C, Chen J, Chen Y et al. $2019 \mathrm{~b}$ ZP2 pathogenic variants cause in vitro fertilization failure and female infertility. Genetics in Medicine 21 431-440. (https://doi. org/10.1038/s41436-018-0064-y)

Escobar SM, Echeverria MO \&Vazquez-Nin GH 2012 Immunohistochemical and ultrastructural visualization of different routes of oocyte elimination in adult rats. European Journal of Histochemistry 56 e17. (https://doi. org/10.4081/ejh.2012.17)

Gook DA, Edgar DH, Borg J \& Martic M 2008 Detection of zona pellucida proteins during human folliculogenesis. Human Reproduction and Embryology 23 394-402. (https://doi.org/10.1093/humrep/dem373)

Gupta SK, Bhandari B, Shrestha A, Biswal BK, Palaniappan C, Malhotra SS \& Gupta N 2012 Mammalian zona pellucida glycoproteins: structure and function during fertilization. Cell and Tissue Research 349 665-678. (https://doi.org/10.1007/s00441-011-1319-y)

Huang HL, Lv C, Zhao YC, Li W, He XM, Li P, Sha AG, Tian X, Papasian CJ, Deng HW et al. 2014 Mutant ZP1 in familial infertility. New England
Journal of Medicine $370 \quad 1220-1226 . \quad$ (https://doi.org/10.1056/ NEJMoa1308851)

Lambert JM, Ashi MO, Srour N, Delpy L \& Sauliere J 2020 Mechanisms and regulation of nonsense-mediated mRNA decay and nonsenseassociated altered splicing in lymphocytes. International Journal of Molecular Sciences 21 1335. (https://doi.org/10.3390/ijms21041335)

Lamas-Toranzo I, Fonseca BN, Querejeta-Fernandez A, IzquierdoRico MJ, Gonzalez-Brusi L, Lorenzo PL, Garcia-Rebollar P, Aviles M \& Bermejo-Alvarez P 2019 ZP4 confers structural properties to the zona pellucida essential for embryo development. eLife 8 e48904. (https://doi. org/10.7554/eLife.48904)

Liu C, Litscher ES, Mortillo S, Sakai Y, Kinloch RA, Stewart CL \& Wassarman PM 1996 Targeted disruption of the mZP3 gene results in production of eggs lacking a zona pellucida and infertility in female mice. PNAS 93 5431-5436. (https://doi.org/10.1073/pnas.93.11.5431)

Liu W, Li K, Bai D, Yin J, Tang Y, Chi F, Zhang L, Wang Y, Pan J, Liang S et al. 2017 Dosage effects of ZP2 and ZP3 heterozygous mutations cause human infertility. Human Genetics 136 975-985. (https://doi. org/10.1007/s00439-017-1822-7)

Pandey AN \& Chaube SK 2014 A moderate increase of hydrogen peroxide level is beneficial for spontaneous resumption of meiosis from diplotene arrest in rat oocytes cultured in vitro. BioResearch Open Access 3 183-191. (https://doi.org/10.1089/biores.2014.0013)

Rankin T, Talbot P, Lee E \& Dean J 1999 Abnormal zonae pellucidae in mice lacking ZP1 result in early embryonic loss. Development 126 3847-3855.

Rankin TL, O'Brien M, Lee E, Wigglesworth K, Eppig J \& Dean J 2001 Defective zonae pellucidae in Zp2-null mice disrupt folliculogenesis, fertility and development. Development 128 1119-1126.

Simon AM, Goodenough DA, Li E \& Paul DL 1997 Female infertility in mice lacking connexin 37. Nature 385 525-529. (https://doi. org/10.1038/385525a0)

Sun L, Fang X, Chen Z, Zhang H, Zhang Z, Zhou P, Xue T, Peng X, Zhu Q, Yin M et al. 2019 Compound heterozygous ZP1 mutations cause empty follicle syndrome in infertile sisters. Human Mutation 40 2001-2006. (https://doi.org/10.1002/humu.23864)

Suzuki H, Togashi M, Adachi J \& Toyoda Y 1995 Developmental ability of zona-free mouse embryos is influenced by cell association at the 4-cell stage. Biology of Reproduction 53 78-83. (https://doi.org/10.1095/ biolreprod53.1.78)

Tiwari M, Prasad S, Tripathi A, Pandey AN, Ali I, Singh AK, Shrivastav TG \& Chaube SK 2015 Apoptosis in mammalian oocytes: a review. Apoptosis 20 1019-1025. (https://doi.org/10.1007/s10495-015-1136-y)

Wang Y, Lv C, Huang HL, Zeng MH, Yi DJ, Tan HJ, Peng TL, Yu WX, Deng HW \& Xiao HM 2019 Influence of mouse defective zona pellucida in folliculogenesis on apoptosis of granulosa cells and developmental competence of oocytesdagger. Biology of Reproduction 101 457-465. (https://doi.org/10.1093/biolre/ioz093)

Wassarman PM 2008 Zona pellucida glycoproteins. Journal of Biological Chemistry 283 24285-24289. (https://doi.org/10.1074/jbc. R800027200)

Wassarman PM \& Litscher ES 2012 Influence of the zona pellucida of the mouse egg on folliculogenesis and fertility. International Journal of Developmental Biology 56 833-839. (https://doi.org/10.1387/ ijdb.120136pw)

Wassarman PM, Qi H \& Litscher ES 1997 Mutant female mice carrying a single mZP3 allele produce eggs with a thin zona pellucida, but reproduce normally. Proceedings Biological Sciences 264 323-328. (https://doi.org/10.1098/rspb.1997.0046)

Zhou Z, Ni C, Wu L, Chen B, Xu Y, Zhang Z, Mu J, Li B, Yan Z, Fu J et al. 2019 Novel mutations in ZP1, ZP2, and ZP3 cause female infertility due to abnormal zona pellucida formation. Human Genetics 138 327-337. (https://doi.org/10.1007/s00439-019-01990-1)

Received 23 January 2020

First decision 14 February 2020

Revised manuscript received 5 June 2020

Accepted 9 June 2020 